\title{
CONTRIBUCIONES DE LA COALICIÓN MULTISECTORIAL PERÚ CONTRA EL CÁNCER
}

\section{CONTRIBUTION OF THE PERUVIAN MULTISECTORIAL COALITION AGAINST CANCER}

\author{
Luis Pinillos-Ashton ${ }^{1, a}$, Abel Limache-García ${ }^{1,2, b}$
}

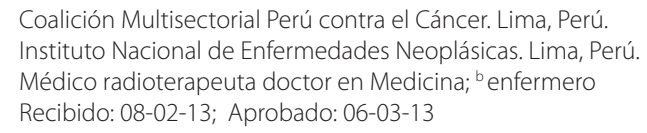

\begin{abstract}
RESUMEN
El cáncer es un problema de salud pública debido a su alta incidencia y mortalidad, su elevado costo social y económico, y por la falta de estrategias intra e intersectoriales, que se evidencia en la falta de acceso a la información y a pruebas de tamizaje. Por todo esto, es necesario enfrentarlo integral y sostenidamente en el tiempo. Luego de un diagnóstico de recursos humanos y materiales a nivel nacional, y de haber observado la actitud de los decisores en salud, se decidió la creación de la Coalición Multisectorial Perú contra el Cáncer con la participación del sector público y no público. Se desarrolló un plan estratégico, y luego un plan nacional para el fortalecimiento de la prevención y control del cáncer. Como resultado, se ha fortalecido el trabajo intersectorial; la promoción de la salud orientada a la prevención del cáncer mediante información, educación y comunicación, se mejoró y acercó la oferta de servicios mediante la desconcentración y descentralización de la atención oncológica.
\end{abstract}

Palabras clave: Neoplasias; Salud pública; Prevención y control; Promoción de la salud (fuente: DeCS BIREME).

\begin{abstract}
Cancer is a public health problem due to its high incidence and mortality, its high social and economic cost, and the lack of intra and intersectorial strategies resulting in failure to access information and screening tests. For this reason, it is necessary to address this problem with a comprehensive and sustainable approach. After performing a diagnosis of the human and material resources at the national level, and after having observed the attitude of healthcare decisors, the Multisector Coalition against Cancer was created with the participation of the public and nonpublic sector. A strategic plan was developed, followed by a national plan for strengthening cancer prevention and control. As a result, intersectorial work and health promotion oriented to cancer prevention through information, education and communication were strengthened. Service supply was improved and optimized through decentralization of oncological care.
\end{abstract}

Key words: Neoplasms; Public health; Prevention and control; Health promotion (source: MeSH NLM).

\section{INTRODUCCIÓN}

El cáncer es un grave y creciente problema de salud pública, por sus elevadas tasas de incidencia y mortalidad, así como su alto costo social y económico, particularmente en países en desarrollo como el Perú. A nivel mundial, en el año 2002, se presentaron aproximadamente 10,9 millones de casos nuevos de cáncer, y 6,7 millones de personas fallecieron por esta misma enfermedad ${ }^{(1)}$. En términos generales, la esperanza de vida de los pacientes a los cinco años es inequitativa entre las diferentes regiones del mundo, variando entre 15 a $60 \%{ }^{(2)}$.
Según estima el Reporte Mundial de Cáncer, en el año 2008 aproximadamente 25 millones de personas vivían con cáncer, se diagnosticaron 12 millones de casos nuevos, y ocurrieron 7 millones de muertes. Para el año 2030 podría esperarse 75 millones de personas con cáncer, 27 millones de casos nuevos de cáncer, y 17 millones de muertes anuales ${ }^{(3)}$.

En la década de 1960 se estimó que el $75 \%$ de los casos de cáncer se diagnosticaban en los países más desarrollados. En la actualidad, la distribución es equitativa, $50 \%$ para los países más desarrollados y 
para los menos desarrollados; se estima que para el año 2030 , si el escenario no se modifica, el $75 \%$ de los casos de cáncer se presentaran en los países menos desarrollados.

En el Perú estamos viviendo un definido incremento de las enfermedades crónicas y degenerativas ${ }^{(4)}$. Se estima en 39305 los casos nuevos de cáncer esperados por año, sin considerar cáncer de piel no melanoma, y según estadísticas internacionales podemos proyectar en algo más de 92245 casos que tenemos hoy día en el Perú ${ }^{(5)}$. De estos, por ser habitualmente diagnosticados tardíamente, menos del 35\% estarán vivos y sin enfermedad a los cinco años, con los costos terapéuticos, sociales y económicos que ello implica ${ }^{(6)}$. Por otro lado, estas cifras no son uniformes en todo el país, pues existe información de un grupo privado que desarrolla un programa integral de control de cáncer que reporta $67 \%$ de sobrevida a cinco años (7), una cifra semejante a la descrita en los estudios SEER y Eurocare.

El problema se agudiza dado que los principales tipos de cáncer que se informan en el Perú son cáncer de cérvix, mama, estómago, próstata y pulmón ${ }^{(5)}$, que afectan a las personas en edades productivas, con el componente social y económico asociado. Estudios de la Agencia de Investigación de Cáncer (IARC) demuestran que, considerando 27 tipos de cáncer en 184 países en 12 regiones del mundo durante el año 2008, el cáncer generó la pérdida de más de 169 millones de años de vida saludables perdidos ${ }^{(8)}$. Además, el cáncer causa la pérdida económica más alta de entre las 15 principales causas de muerte en el mundo (895 billones de dólares), siendo $20 \%$ más alta que las enfermedades cardiovasculares (753 billones de dólares) ${ }^{(9)}$. El impacto de las muertes prematuras y de la discapacidad a nivel mundial en el año 2008 fue de 895 billones de dólares ( $1,5 \%$ del Producto Bruto Mundial), la cual no incluye los costos de tratamiento de cáncer.

En el Perú, sin considerar los costos terapéuticos, el cáncer ocasiona la pérdida de aproximadamente 378 mil años de vida saludables (AVISA) equivalente a cerca de 900 millones de dólares por año, calculado sobre la base del salario mínimo nacional. Los tipos de cáncer que ocasionaron mayor número de años de vida saludables perdidos $y$, por ende, mayor pérdida económica fueron, el cáncer de estómago, de cérvix, de mama, las leucemias, de hígado y de pulmón (10).

Lo señalado muestra solo unas de las razones por la que el cáncer representa un grave problema de salud pública; sin embargo, es un problema no solo del sector salud sino también de todos los sectores del
Estado, e inclusive de las organizaciones privadas y no gubernamentales.

\section{AGRAVANTES DEL PROBLEMA}

Si no consideramos al cáncer como un tema que necesita decisión política para un control integral, con una propuesta inclusiva y sostenida, seguirá siendo un problema de salud pública. En ese sentido, la ausencia o presencia de múltiples situaciones podrían agravar la no mejora de la campaña contra el cáncer. Entre dichas situaciones tenemos:

Falta de acceso a la información preventiva. Se evidencia en la poca participación de los medios de comunicación en la transmisión de mensajes preventivos, o espacios destinados a fomentar un estilo de vida saludable en el que se incluya, sobre todo, información acerca de los daños ocasionados por el consumo directo o indirecto de tabaco; los daños de una alimentación basada en productos procesados, grasas saturadas o, en general, "alimentos chatarra", y mensajes que informen la relación de agentes infecciosos (el papiloma virus humano, el Helicobacter pilory, o el virus de la hepatitis) con el desarrollo del cáncer de cérvix, estómago e hígado.

\section{Dificultades de acceso a los servicios especializados} de manejo del cáncer. Nuestro accidentado territorio; la sofisticación de los equipos humanos y materiales necesarios para un manejo especializado del cáncer, y el centralismo en Lima y las grandes capitales, donde se concentran las inversiones para el manejo del cáncer, hacen que los recursos no se encuentren disponibles para la mayoría de los peruanos; hacen necesaria una política concertada multisectorial a fin de acercar los servicios especializados por niveles para cubrir las necesidades de la población. En el año 2005 se calculó la aparición de 39305 casos nuevos de cáncer y se estima que solo 13000 peruanos se atendieron en algún servicio médico oncológico, cifra deducida considerando que solo en el Instituto Nacional de Enfermedades Neoplásicas (INEN), hasta ese momento el único instituto especializado en cáncer en el sistema de salud pública, se diagnosticaron ese año, 8745 casos ${ }^{(11)}$, es decir, solo uno de cada tres o cuatro peruanos con cáncer lograba ser diagnosticado bajo este sistema.

Enfoque terapéutico y no preventivo. Es un problema debido a que muchos consideran que el tratamiento, y más aun el tratamiento sofisticado y costoso, es la mejor forma de enfrentar el cáncer, particularmente medido por el acceso a medicamentos 
biológicos de última generación y equipos sofisticados, cuando la estrategia fundamental debe de ser integral, con énfasis en la prevención, detección y diagnóstico temprano para tratarlo con oferta de cirugía, radioterapia, complementado por especialistas en tratamientos de quimioterapia y con acceso a cuidados paliativos. El cáncer no es una sentencia de muerte, existen medios demostrados para prevenirlo, tratarlo y curarlo. Un tercio de todos los cánceres pueden prevenirse y un tercio puede curarse con éxito ${ }^{(12,13)}$. El cáncer es la única enfermedad crónica que al ser detectada y diagnosticada a tiempo, se cura; sin embargo, en nuestro país, el $75 \%$ de los casos tiene la enfermedad avanzada, así el año 2005 se registraron 18000 muertos.

Falta de médicos especializados. Es un grave problema la falta profesionales para atender la demanda nacional con especialistas en la atención del cáncer en sus diferentes modalidades de tratamiento. Considerando la carga de cáncer en nuestro país, se necesitarían no menos de 80 equipos de radioterapia que implicaría, por lo menos, 120 radioterapeutas; sin embargo, contamos solo con 20 equipos de radioterapia y la mayoría de ellos centralizados en Lima, mientras que cada año egresan aproximadamente cinco radioterapeutas; así mismo, contamos aproximadamente con 450 cirujanos oncólogos pero se requieren no menos de 500 más; se cuenta con 80 oncólogos médicos y necesitamos, por lo menos, 300 .

Necesidad de gobierno multisectorial. Porque el problema concierne a todos los sectores del Estado; en consecuencia, el control del cáncer precisa del actuar de todos los sectores, necesita de intervenciones que vinculen y comprometan a los poderes del Estado, como el Legislativo, el Ejecutivo con sus ministerios de Economía, Educación, Agricultura, Industria, Minería, Trabajo y Promoción del Empleo, Ministerio de la Mujer entre otras, y que las intervenciones que se implementen involucren también el compromiso de los gobiernos regionales y locales. Estos niveles de gobierno tienen responsabilidad en el impulso de factores protectores contra el cáncer, como por ejemplo en la formación de alumnos responsables en el cuidado de su salud; en el impulsar estrategias que permitan a la población un mayor consumo de frutas y vegetales en todas las regiones del país; en acciones que permitan que la población no solo esté informada de los beneficios de la actividad física, sino además, cuente con los espacios y condiciones necesarias para hacerlo. Estos estamentos, además de asumir su rol según niveles de gobierno y responsabilidades institucionales, deben legislar y hacer que se cumplan las leyes que promuevan la salud y controlen los factores de riesgo.
Problemas en la legislación peruana. La historia de nuestra legislación para el control del tabaquismo es explicativa del problema. El año 1991 se promulga la Ley 25357, y no es hasta el 2004 en el que el Perú ratifica el Convenio Marco de Control del Tabaco promovida por la OMS comprometiéndose a adecuar su legislación a lo establecido por el mencionado convenio; sin embargo, nuestro país tuvo que esperar dos años para que el Legislativo promulgue la Ley 28705, Ley para la Prevención y Control de los Riesgos del Consumo de Tabaco (6 de abril de 2006), y dos años más para que se apruebe el reglamento de la mencionada Ley (5 de julio de 2008), pero el tabaquismo y el cáncer seguirían siendo problema de salud pública, dado que la Ley 28705 no contemplaba lo establecido por el Convenio Marco de la OMS, por lo que cuatro años después de haber sido promulgada la Ley, se modifica mediante Ley 29517 (31 de marzo de 2010). Actualmente, es responsabilidad de los gobiernos locales su aplicación y vigilancia ${ }^{(14)}$.

\section{LA COALICIÓN MULTISECTORIAL PERÚ CONTRA EL CÁNCER}

En un esfuerzo para superar la situación presentada, se convocó a todos las instituciones interesadas o comprometidas con el tema para discutir la problemática y plantear conjuntamente una estrategia para enfrentar coordinadamente el cáncer en el país, compartir ideas, recursos humanos y materiales, a fin de acercarnos a la población. Así nació la Coalición Multisectorial Perú contra el Cáncer en el año 2004.

El proyecto que buscó crear la Coalición inició el año 2002, cuando se revisó la historia de los planes de control nacionales de cáncer y se realizó una encuesta a nivel nacional de todos los servicios de salud y hospitales para identificar fortalezas profesionales, funcionabilidad de equipos para el manejo del cáncer, y el nivel de utilización de ellos.

La encuesta nos mostró que se tenía profesionales especializados en cáncer, con funciones médicas o de enfermería generales ${ }^{(15)}$. Además, se mostró que algunos hospitales contaban con mamógrafos pero no con mamografistas y en otros, mamógrafos que no funcionaban por falta de reactivos o de tecnólogos.

Se identificó como un gran problema la falta de acceso a la información, tanto del público en general como de los profesionales. Por otro lado, se observó la falta de acceso a las pruebas de tamizaje, que no había control de calidad de ellos y que no habían centros especializados, lo cual concentraba la atención de los pacientes en el INEN, que ya se encontraba saturado, 
y en los grandes hospitales del seguro, donde el tiempo de espera es muy largo.

Se estudiaron las estadísticas regionales y hospitalarias, se extendió las estrategias que cada una tenía con relación al cáncer, y se planificó e inició con estos datos un plan de desconcentración y descentralización de la atención oncológica.

Considerando este diagnóstico y con el apoyo de la Sociedad Americana del Cáncer y el INEN, se convocó a todas las instituciones interesadas en el tema y se encontró una gran receptividad y deseo de colaborar de todos los sectores y niveles de gobierno, con lo que se constituyó la Coalición Multisectorial Perú contra el Cáncer, con una filosofía inclusiva y participativa, con una visión para el año 2016 de tener un Perú libre de cáncer avanzado, con cultura y educación en salud, y con acceso a servicios de calidad para el control del cáncer que promueva cambios importantes en la política nacional, priorizando acciones de prevención y promoción de la salud facilitando el acceso a los servicios oncológicos ${ }^{(16)}$.

Los participantes iniciales de la Coalición Multisectorial Perú contra el Cáncer fueron: la Comisión de Lucha Antitabaquica (COLAT); la Sanidad de la Policía Nacional del Perú; el seguro social (EsSalud); la Fundación Peruana de Cáncer; el INEN; el Instituto Nacional de Salud; el Ministerio de Salud, la Municipalidad de San Borja; el Colegio de Enfermeros del Perú; la Sociedad Peruana de Cancerología; la Sociedad de Oncología Médica; la Dirección Nacional de la Fuerza Aérea; la Sociedad Americana del Cáncer, y el Instituto de Cáncer de Washington; con todos ellos se elaboró el "Plan Estratégico Perú 2006-2016, Perú contra el Cáncer" sobre el cual se hace un diagnóstico situacional; se identifica las necesidades de acceso, se define el compromiso de las instituciones; se define la visión y misión y los ocho lineamientos estratégicos, y concluye que se debe de elaborar e implementar un Plan Nacional de Prevención y Control del Cáncer dentro del marco normativo y jurídico del MINSA y del Estado.

Más adelante se sumaron las siguientes instituciones: la Municipalidad del Callao; la Municipalidad de Carmen de la Legua; la Municipalidad de Miraflores; los gobiernos regionales de Lambayeque, La Libertad, Ayacucho, Arequipa, Loreto y Callao; la Sociedad de Enfermeras Oncólogas; la Asociación de Pacientes; el voluntariado; el Patronato de Lucha contra el Cáncer de Huancayo; el Colegio de Obstetras; la Comisión Nacional para el Desarrollo y Vida sin Drogas, DEVIDA; el Hospital Militar Central; el Colegio de Psicólogos del Perú; el Hospital Regional Honorio Delgado de Arequipa; el Colegio Odontológico del Perú; el Instituto Regional de
Tabla 1. Ejes y objetivos estratégicos de la Coalición Multisectorial Perú contra el Cáncer.

\begin{tabular}{ll}
\hline Eje estratégico & Objetivo estratégico \\
\hline $\begin{array}{l}\text { Promoción y } \\
\text { prevención }\end{array}$ & $\begin{array}{l}\text { Fortalecer la prevención primaria, } \\
\text { secundaria y terciaria con énfasis } \\
\text { en la detección y manejo temprano. }\end{array}$ \\
\hline Normatividad & $\begin{array}{l}\text { Velar por el cumplimiento de } \\
\text { normas existentes y crear normas } \\
\text { necesarias. }\end{array}$ \\
\hline Acceso & $\begin{array}{l}\text { Fortalecer la desconcentración y } \\
\text { descentralización de la atención } \\
\text { oncológica }\end{array}$ \\
\hline $\begin{array}{l}\text { Vigilancia } \\
\text { epidemiológica activa } \\
\text { e investigación }\end{array}$ & $\begin{array}{l}\text { Implementar la } \\
\text { epidemiológica activa y promover la } \\
\text { investigación }\end{array}$ \\
\hline Grupos organizados & $\begin{array}{l}\text { Favorecer la conformación de } \\
\text { grupos de pacientes }\end{array}$ \\
\hline Voluntariado & $\begin{array}{l}\text { Apoyar acciones del voluntariado } \\
\text { Captar recursos financieros, } \\
\text { Desarrollo de } \\
\text { recursos }\end{array}$ \\
\hline $\begin{array}{l}\text { Información y } \\
\text { comunicación }\end{array}$ & $\begin{array}{l}\text { Crear el programa de educación y } \\
\text { comunicación. }\end{array}$ \\
\hline
\end{tabular}

Enfermedades Neoplásicas Norte (IREN); el IREN Sur, el Colegio Médico del Perú; la Liga de Lucha contra el Cáncer; la Asociación de Laringectomizados del Perú, y el Colegio de Nutricionistas.

Aprobado el Plan Estratégico se procedió a la elaboración del Plan Nacional para el Fortalecimiento de la Prevención y Control del Cáncer en el Perú, cuyo objetivo general es el de contribuir a la reducción de la incidencia, morbilidad y mortalidad del cáncer, y mejorar la calidad de vida de los pacientes oncológicos en el Perú, aplicando intervenciones sistemáticas basadas en evidencias científicas para la promoción de la salud, prevención, detección temprana, diagnóstico, tratamiento, rehabilitación y cuidados paliativos, con un enfoque integral para contar con personas saludables, cada uno de los objetivos específicos del Plan Nacional corresponde a los objetivos estratégicos que figuran en la Tabla 1.

\section{AVANCE DE RESULTADOS DE LA COALICIÓN}

Desde su implementación, la Coalición ha elaborado las siguientes guías y normas:

- Guía para la promoción de la salud orientada a la prevención del cáncer.

- Norma técnico - oncológica para la prevención, detección y manejo de las lesiones premalignas del cuello uterino a nivel nacional (RJ 121-RJ- INEN-2008). 
- Norma técnico - oncológica para la prevención, detección y diagnóstico temprano del cáncer de mama a nivel nacional (RJ 120-RJ- INEN-2008).

Al haberse desarrollado estas guías y normas con participación multisectorial, han sido de más fácil aceptación; además dichos documentos son socializados en talleres con participación de profesionales de la salud y educación regular de las diferentes regiones del país, para conocer su contenido y uso.

En el tema de educación, se han capacitado más de 10000 profesionales de la salud, entre médicos, enfermeras y obstetrices, más de 4000 docentes de educación regular y cerca de 2000 promotores de salud.

En educación pública se han desarrollado actividades en el marco de la "Semana Perú Contra el Cáncer" (RM 710-2003-SA/DM), marchas, ferias y concursos gastronómicos saludables, foros, talleres, publicidad en prensa escrita y televisiva a nivel nacional.

En relación al cáncer de cérvix, se ha fortalecido la capacitación de profesionales en estrategias de "ver y tratar" mediante el uso de pruebas de tamizaje y tratamiento de lesiones preneoplásicas tales como la inspección visual con ácido acético y la crioterapia; experiencia que fue replicada en otros países como Bolivia, Colombia y Nicaragua en coordinación con agencias cooperantes. En nuestro país se han entregado equipos luego de la capacitación.

En relación al cáncer de mama, se inició una campaña publicitaria muy fuerte "A las mujeres no nos gana el cáncer de mama", difundiendo mucha información en paneles y anuncios publicitarios en televisión. Esta campaña informativa tiene un impacto mayor en las zonas urbanas por lo que se realiza la difusión por radios locales y regionales, con mensajes adecuados culturalmente para poblaciones alejadas.

En relación a la nutrición, se ha desarrollado cursos y ferias en las diferentes instituciones de salud como en el Instituto Regional de Enfermedades Neoplásicas de Trujillo y Arequipa.

En cuanto al control del tabaco, junto a la COLAT, institución participante activa de la Coalición, se ha logrado leyes importantes:

- Ley 28705 Ley General para la Prevención y control de los Riesgos del Consumo de Tabaco (12 de abril 2006).

- Reglamento de la Ley 28705 Ley General para la Prevención y control de los Riesgos del Consumo de Tabaco (4 de julio 2008).
- Ley 29517 Ley que modifica la Ley 28705 Ley General para la Prevención y control de los Riesgos del Consumo de Tabaco para adecuarse al Convenio Marco de la Organización Mundial de la Salud (OMS) para el control del Tabaco (31 de marzo 2010).

Diversas publicaciones internacionales precisan la importancia de la actividad física junto con la alimentación rica en frutas y verduras ${ }^{(17)}$, así como la práctica de estilos de vida saludables para la prevención e incluso para la recuperación de los pacientes tratados ${ }^{(18)}$. Esta información ha sido difundida mediante paneles, programas de televisión y radio, como parte de las estrategias de prevención primaria y secundaria

En los siete años de funcionamiento, la Coalición Multisectorial Perú contra el Cáncer, por acción de sus integrantes, puede presentar resultados que confirman que es el camino correcto, prueba de ello es que antes 1 de cada 3 o 4 peruanos se diagnosticaban en el sistema público de atención, hoy se atiende a 1 de cada 2; estimación que es el resultado del incremento en el número de atendidos en el INEN, al 2012 este instituto diagnostica más de 10000 pacientes ${ }^{(11)}$; en tanto, en el IREN Norte ${ }^{(19)}$ el 2011 se atendieron 1495 pacientes, y en el IREN Sur 1922 pacientes ${ }^{(20)}$. Considerando, además, que tenemos diez preventorios en funcionamiento, diez unidades oncológicas en hospitales nacionales, el doble de equipos de radioterapia, así como mamógrafos de calidad, y en mayor número.

\section{REFLEXIONES FINALES}

El cáncer tiene un origen multifactorial con ciertas variables inmodificables como la carga genética, pero con muchas otras modificables a través de nuestros estilos de vida. El cáncer a nivel mundial causa mayor número de muertes que enfermedades como la tuberculosis, el $\mathrm{VIH}$ y el paludismo, individual y colectivamente, y el número de víctimas está incrementándose rápidamente. A menos que adoptemos medidas urgentes para el 2030 , más de 13 millones de personas morirán de cáncer cada año, la mayoría de ellas en países en vías de desarrollo como el nuestro.

El Perú debe de enfocar, coordinada y estratégicamente, el problema del cáncer con un proyecto multisectorial y multiinstitucional coordinado y manejado por especialistas de las distintas instituciones siguiendo la normatividad que dicta el INEN, responsable del cáncer en el país por Ley, manteniendo un solo programa sustentado en el tiempo, mejorándolo, pero no reinventándolo.

Se requiere de un programa integral, sostenido en el tiempo, con objetivos de corto, mediano y largo plazo, 
que vaya de la prevención a los cuidados paliativos; un programa que contemple educación; equipamiento para diagnóstico de lesiones premalignas, o malignas tempranas; centros especializados para su manejo por especialistas, y evaluación constante epidemiológica y estadística de la marcha de los programas, y su impacto sobre la salud nacional. Requiere tecnología altamente especializada, estratégica y profesionalmente seleccionada, así como de la formación de personal calificado para los distintos niveles de atención y, finalmente, requiere participación activa de la comunidad para lograr el objetivo de salud para todos pero por todos.

Contribuciones de autoría: LPA y ALG han participado en la concepción y diseño del artículo, recolección, obtención de resultados, análisis e interpretación de datos y redacción del artículo.

\section{REFERENCIAS BIBLIOGRÁFICAS}

1. Ferlay J, Bray F, Pisani P, Parkin DM: GLOBOCAN 2002: Cancer Incidence, Mortality and Prevalence Worldwide. Lyon, Francia: IARCPress; 2004.

2. Coleman MP, Quaresma M, Berrino F, Lutz JM, De Angelis R, Capocaccia R, et al. Cancer survival in five continents: a worldwide population-based study (CONCORD). Lancet Oncol. 2008;9(8):730-6.

3. Boyle P, Levin B (ed). World Cancer Report 2008. International Agency for Research on Cancer. Lyon: OMS; 2008.

4. Perú, Dirección Ejecutiva de Enfermedades No Transmisibles, Oficina General de Epidemiología. Análisis de la Situación de la Salud. Lima: MINSA; 1999.

5. International Agency for Research on Cancer. GLOBOCAN 2008 [Internet]. Lyon: OMS; c2010 [citado el 14 de enero de 2013]. Disponible en: http://globocan.iarc.fr/

6. Sankaranarayanan $\mathrm{R}$, Swaminathan $\mathrm{R}$ (ed). Cancer survival in Africa, Asia, the Caribbean and Central America. Lyon: WHO; 2011.

7. Instituciones y Organizaciones públicas y privadas: Oncosalud. En: Academia Peruana de Salud. Historia de la Salud en el Perú. Volumen 25. Lima: Academia Peruana de Salud; 2011.

8. Soerjomataram I, Lortet-Tieulent J, Ferlay J, Forman D, Mathers C, Parkin
DM, et al. Estimating and validating disability-adjusted life years at the global level: a methodological framework for cancer. BMC Med Res Methodol. 2012;12:125.

9. American Cancer Society. The global economic cost of cancer. Atlanta: American Cancer Society; 2010

10. Perú, Ministerio de Salud. La Carga de Enfermedad y Lesiones en el Perú. 1era Ed. Lima: MINSA; 2009

11. Perú, Instituto Nacional de Enfermedades Neoplásicas. Datos estadísticos [Internet]. Lima: INEN; 2013 [citado el 14 de enero de 2013]. Disponible en: http://www.inen. sld.pe/portal/documentos/pdf/estadistica/ datos_estadisticos/20052011_INEN NM_FRECUENTES_2000_2009_vf.pdf)

12. International Atomic Energy Agency (IAEA). Fighting Cancer in the Developing World [Internet]. Vienna: IAEA; c19982012 [citado el 14 de enero de 2013]. Disponible en: http://www.iaea.org/ newscenter/news/2012/fightingcancer. html

13. Organización Mundial de la Salud. Control del Cáncer. Aplicación de los conocimientos. Ginebra: OMS; 2007.

14. Historia del Tabaquismo en el Perú. En: Academia Peruana de Salud. Historia de la Salud en el Perú. Volumen 25. Lima: Academia Peruana de Salud; 2011.
15. Coalición Multisectorial Perú contra el Cáncer. Plan Nacional para el Fortalecimiento de la Prevención y Control del Cáncer en el Perú. Lima: INEN; 2008.

16. Coalición Multisectorial Perú contra el Cáncer. Plan Estratégico 2006 - 2016. Lima: OPS; 2006.

17. Anand P, Kunnumakkara AB, Sundaram C, Harikumar KB, Tharakan ST, Lai OS, et al. Cancer is a preventable disease that requires major lifestyle changes. Pharm Res. 2008;25(9):2097-116.

18. Kruk J. Physical Physical activity in the prevention of the most frequent chronic diseases: an analysis of the recent evidence. Asian Pac J Cancer Prev. 2007;8(3):325-38.

19. Instituto Regional de Enfermedades Neoplásicas del Norte [Internet]. La Libertad: MINSA; [citado el 12 de diciembre del 2012]. Disponible en: http://www.irennorte.gob.pe/

20. Instituto Regional de Enfermedades Neoplásicas del Sur [Internet]. Arequipa: MINSA; [citado el 12 de diciembre del 2012]. Disponible en: http://www.irensur. gob.pe/web/

Correspondencia: Luis Pinillos Ashton

Dirección: Av. Paseo de la República 3650. Lima 27, Perú.

Teléfono: (511) 4225520

Correo electrónico:luispinillos@radioncologia.com 